\title{
OCEANUS: A high science return Uranus orbiter with a low-cost instrument suite
}

C. M. Elder ${ }^{\mathrm{a},}$, , A. M. Bramson ${ }^{\mathrm{b}}$, L. W. Blum ${ }^{\mathrm{c}}$, H. T. Chilton ${ }^{\mathrm{d}}$, A. Chopra ${ }^{\mathrm{e}}$, C. Chu ${ }^{\mathrm{f}}$, A. Das ${ }^{\mathrm{g}}$, A. B. Davis ${ }^{\mathrm{h}}$, A. Delgado ${ }^{\mathrm{i}}$, J. Fulton ${ }^{\mathrm{h}}$, L. M. Jozwiak ${ }^{\mathrm{j}}$, A. Khayat ${ }^{\mathrm{c}}$,, M. E. Landis ${ }^{\mathrm{b}}$, J. L. Molaro ${ }^{\mathrm{a}}$, M. Slipski $^{\text {h }}$ S. Valencia ${ }^{1}$, J. Watkins ${ }^{\mathrm{m}+}$, C. L. Young ${ }^{\mathrm{n}}$, C. J. Budney ${ }^{\mathrm{a}}$, K. L. Mitchell ${ }^{\mathrm{a}}$

${ }^{a}$ Jet Propulsion Laboratory, California Institute of Technology, Pasadena, CA 91109, United States

${ }^{\mathrm{b}}$ Lunar and Planetary Laboratory, University of Arizona

${ }^{\mathrm{c}}$ NASA/Goddard Space Flight Center

${ }^{\mathrm{d}}$ Georgia Institute of Technology

${ }^{\mathrm{e}}$ Australian National University

${ }^{\mathrm{f}}$ Embry-Riddle Aeronautical University

g Purdue University

${ }^{\mathrm{h}}$ University of Colorado at Boulder

${ }^{i}$ NASA/Stennis Space Center

j Johns Hopkins University/Applied Physics Laboratory

${ }^{\mathrm{k}}$ University of Maryland, College Park

${ }^{1}$ Washington University in St. Louis

${ }^{m}$ California Institute of Technology

${ }^{n}$ NASA Langley Research Center

${ }^{+}$now at NASA Johnson Space Center

*Corresponding author: Tel.: +1 8183549381

E-mail address: Catherine.Elder@jpl.nasa.gov (C. M. Elder)

Address: Jet Propulsion Laboratory

$\mathrm{M} / \mathrm{S} 183-301$

4800 Oak Grove Drive

Pasadena, CA 91109

\begin{abstract}
Ice-giant-sized planets are the most common type of observed exoplanet, yet the two ice giants in our own solar system (Uranus and Neptune) are the least explored class of planet, having only been observed through ground-based observations and a single flyby each by Voyager 2 approximately 30 years ago. These single flybys were unable to characterize the spatial and temporal variability in ice giant magnetospheres, some of the most odd and intriguing magnetospheres in the solar system. They also offered only limited constraints on the internal structure of ice giants; understanding the internal structure of a planet is important for understanding its formation and evolution. The most recent planetary science Decadal Survey by the U.S. National Academy of Sciences, "Vision and Voyages for Planetary Science in the Decade 2013 - 2022," identified the ice giant Uranus as the third highest priority for a Flagship mission in the decade 2013-2022. However, in the event that NASA or another space agency is unable to fly a Flagship-class mission to an ice giant in the next decade, this paper presents a mission concept for a focused, lower cost Uranus orbiter called OCEANUS (Origins and Composition of the Exoplanet Analog Uranus System). OCEANUS would increase our understanding of the interior structure of Uranus, its magnetosphere, and how its magnetic field is generated. These goals could be achieved with just a magnetometer and the spacecraft's radio
\end{abstract}


system. This study shows that several of the objectives outlined by the Decadal Survey, including one of the two identified as highest priority, are within reach for a New-Frontiers-class mission.

Keywords: Uranus, Ice Giant, New Frontiers, mission concept

\section{Introduction}

The ice giants are the most poorly characterized class of planets in our solar system; despite the tremendous scientific insights from ground-based observations and the solitary Voyager 2 flyby, the ice giants still remain largely unexplored due to a dearth of spacecraft missions dedicated to studying them. The knowledge that we do have reveals that ice giants differ substantially from gas giants - the more thoroughly explored Jupiter and Saturn cannot be used as analogs for Uranus and Neptune. Unlike gas giants, which are composed primarily of hydrogen and helium, ice giants are thought to be composed primarily of ices and rocks [1]. However, the phases, distributions, and exact compositions of these ices and rocks are unknown [1]. The magnetic fields of ice giants differ substantially from those of Jupiter and Saturn as well [2]. Voyager data suggest that both Uranus and Neptune have magnetic fields with strong quadrupole moments and dipole axes that are offset from their spin axes by large angles [2]. Dynamo theory and expected conductivities suggest that fields such as these could be generated in an ionic ocean with strong currents, but the depth, extent, and conductivity of this ocean are uncertain [2]. Furthermore, understanding the stages and location of formation of the ice giants is critical to understanding solar system formation and evolution [e.g. 3, 4]. Measuring the noble gas abundances and isotope ratios in an ice giant could provide insight into the conditions under which the planet formed [e.g. 5].

A return to the ice giants is perhaps more important now than ever before, because NASA's Kepler mission found that ice giant-sized planets are the most common type of observed exoplanet [6]. While observational biases are expected to underreport terrestrial planets, it is striking that ice-giant-sized planets are more common than gas-giant-sized planets. Kepler has also discovered many super-Earths, which are larger than Earth but smaller than Uranus or Neptune $\left(\sim 4 \mathrm{R}_{\oplus}\right)$ [6]. Empirical observations indicate that "super-Earth" planets larger than $1.6 \mathrm{R}_{\oplus}$ are too low density to be composed of iron and silicates alone [7], suggesting the ice giants in our solar system are the closest analog for this newly discovered class of planet as well.

A mission to either Uranus or Neptune could greatly improve our understanding of ice giant planets, but the distance to these planets makes a mission to either of them challenging. Of the two ice giants, Uranus is closer to Earth, and therefore generally more accessible than Neptune; the shorter cruise time has the additional benefit of decreasing the longevity required of the instruments and any radioisotope power system that such a mission might use, such as MultiMission Radioisotope Thermoelectric Generators (MMRTGs). We chose to develop a mission concept to Uranus rather than to Neptune, both because of the accessibility and because such a mission would enable us to study the unique effects of Uranus' extreme axial tilt $\left(98^{\circ}\right)$. This tilt leads to intense seasonal changes, which affect the intensity of atmospheric dynamics [8], the insolation conditions of the satellites, and the interactions between the magnetosphere and the solar wind. Voyager 2 flew by Uranus in 1986, one year after southern summer solstice. To investigate seasonal changes through comparison with Voyager data, the next mission to Uranus should arrive closer to equinox than solstice; the next equinox will occur in 2049 and Uranus' next southern summer solstice will occur in 2070 at which time insolation conditions will be the 
same as during Voyager's flyby.

"Vision and Voyages for Planetary Science in the Decade 2013 - 2022" [9] (hereafter

Decadal Survey) suggests that a mission to an ice giant should be the third highest priority for a Flagship mission (the NASA planetary science mission class with the highest budget cap). Most previous ice giant mission concepts have focused on Flagship-class missions because of the challenges involved in reaching the ice giants and the large number of outstanding science questions. However, here, we show that a less expensive mission concept, of the scale of a New Frontiers mission, is capable of addressing many of the open scientific questions about the ice giants. This mission concept, named OCEANUS (Origins and Composition of the Exoplanet Analog Uranus System), would employ a small instrument suite onboard a spacecraft that would orbit Uranus.

OCEANUS is the result of the 2016 Planetary Science Summer School (PSSS) hosted by the Jet Propulsion Laboratory (JPL), California Institute of Technology, which aims to offer participants an authentic but primarily educational experience of the mission proposal process [10]. The concept mission was developed through a ten-week-long program of weekly teleconferences, which culminated in a week of intense design work at JPL and included three sessions working with JPL's Team X. Here we present the results from this summer school: a small instrument suite can address high priority science objectives for Uranus.

\section{Science Objectives}

Both the U.S. Decadal Survey and a white paper for the European Space Agency (ESA) have identified a similar set of scientific objectives for a mission to Uranus [9,11,12]. We include the text of these science objectives as an appendix. A recent NASA ice-giant mission study reevaluated science priorities and Flagship-class mission concepts in preparation for NASA's third Planetary Science Decadal Survey [13]. They identified twelve science objectives, which are consistent with those identified by the Decadal Survey.

In this study, we explore the possibility that a lower-cost mission than previous studies $[9,11,12$, or 13$]$ could address a significant fraction of the high-priority questions about Uranus. Our goal was to maximize science return while minimizing instrument cost to assess the feasibility of a lower-cost mission. This can be achieved by focusing on the radial distribution of mass in Uranus' interior and the structure of Uranus' magnetosphere, with implications for understanding dynamo generation in an ionic ocean. OCEANUS' detailed science and measurement objectives are shown in Table 1. With a simple instrument suite of a magnetometer and a radio science system, OCEANUS would achieve Decadal Survey objectives 2, 4, and 7 [9] and address or partially address ESA white paper questions $1.1,1.3,1.4,1.5,1.6,3.1$, and 3.3 $[11,12]$. OCEANUS' magnetosphere objectives are also responsive to Key Science Goals 3 and 4 in the Solar and Space Physics Decadal Survey whose Solar Wind-Magnetosphere Interactions Panel identified a Uranus orbiter as their highest priority in planetary magnetospheres [14]. Optionally including an atmospheric probe ${ }^{1}$ would enable the determination of the atmospheric structure at the probe descent location and the bulk noble gas abundances and isotopic ratios (Decadal Survey objective 3; ESA white paper question 1.1). Through both the probe and occultation observations, OCEANUS would also make progress towards determining the vertical

\footnotetext{
${ }^{1}$ For the purposes of the educational exercise, we assumed that OCEANUS would have a probe donated as furnished equipment. In reality, this could be an opportunity for international collaboration (see section 4.3).
} 
profile of zonal winds and condensable materials in Uranus' atmosphere (Decadal Survey objective 9). Achieving OCEANUS' science objectives would greatly increase our understanding of ice giants, which demonstrates that a New-Frontiers-class mission to Uranus is feasible and scientifically compelling, responding (without a probe) to 4 of the 9 objectives identified in the Decadal Survey and 7 out of 17 questions posed by the ESA white paper.

Table 1: OCEANUS's science objectives, measurement objectives, and the instruments necessary to achieve these objectives. The first column indicates the U.S. National Academies Decadal Survey and ESA White Paper Objectives that OCEANUS would address (without a probe: OCEANUS would respond to 4 out of 9 Decadal Survey objectives and 7 out of 17 ESA white paper questions).

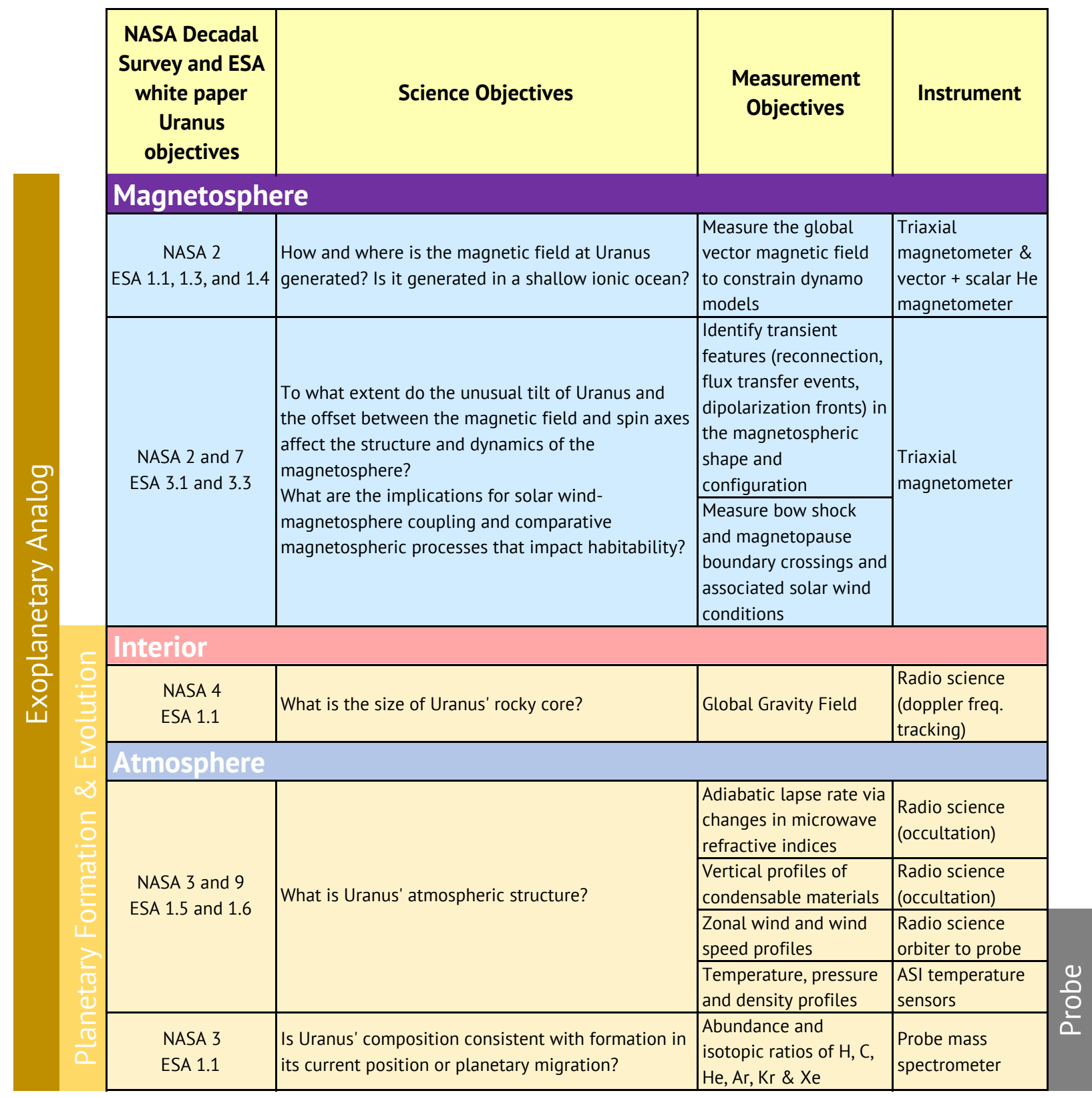

\section{Mission Concept Overview}

The OCEANUS mission architecture was designed using the concurrent systems 
engineering approach of JPL's Team X, which includes real-time trades between mass, power, volume, and cost $[15,16]$. We worked with JPL's Team X for three three-hour sessions to identify an architecture able to achieve all of the science objectives described in Table 1 . The distance between Earth and the ice giants presents the biggest challenge for achieving this mission. In particular, opportunities for a Jupiter gravity assist to Uranus (or Neptune) are rare in the next two decades, so OCEANUS would likely need to rely on Earth and Venus gravity assists and Solar Electric Propulsion (SEP), which tends to increase the cost of the mission. In this concept study, we have focused on one such trajectory that would launch in August 2030 on an Atlas V 551 rocket and arrive at Uranus in 2041 (Figure 1). This trajectory would include two Venus gravity assists and one Earth gravity assist; leveraging SEP for a seven-year period, until a solar distance of 1.5 AU is achieved. Following this phase, the spacecraft begins a four-year safe hibernation state, which would last until the final approach to Uranus.

If included, the probe would be released 30 days before orbit insertion. It would start collecting data at 0.1 bars and would return data for up to 1 hour. The atmospheric structure instrument is sensitive up to a temperature of $500 \mathrm{~K}$ and a pressure of 5 bars. However, OCEANUS' communication frequencies would be absorbed by the methane layer in Uranus' atmosphere, so although the probe is likely to survive to lower layers in Uranus' atmosphere, it would not be able to communicate with the orbiter below the methane layer which is expected to occur somewhere between 1 and 3 bars [17]. However, there is still some uncertainty about the distribution, circulation, and seasonal variability of methane in Uranus' atmosphere [18-20], so the depth to which we would receive data cannot be known precisely a priori. During the probe science phase, the orbiter would use the high gain antenna to receive data from the probe, and we would track the orbiter from Earth with the low gain antennae. The data from the probe would be stored on the orbiter and subsequently relayed back to Earth. Uranus orbit insertion would occur two hours after probe entry. The proximity of these two mission critical events is one of the major risks of OCEANUS; however, Galileo's planned trajectory included communication with the probe for up to 75 minutes and orbit insertion 65 minutes after the end of probe relay [21]. In practice, the Galileo probe stopped function 61.4 minutes after entry [22] and a successful Jupiter orbit insertion started 82 minutes later [23]; for OCEANUS, Uranus orbit insertion would occur at least one hour after losing the signal from the probe. 


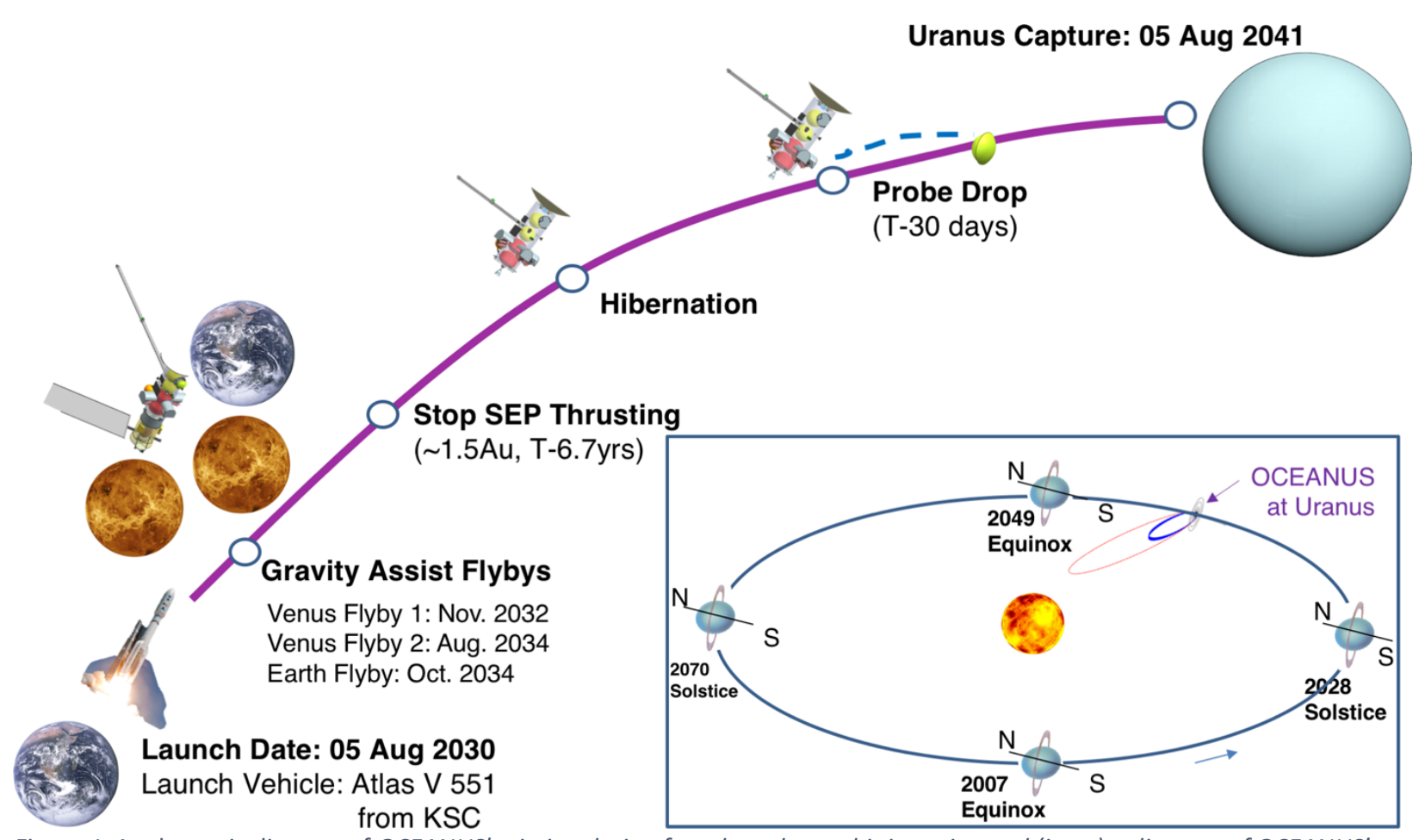

Figure 1: A schematic diagram of OCEANUS' mission design from launch to orbit insertion and (inset) a diagram of OCEANUS' arrival relative to Uranus' orbit. The red ellipse indicates the capture orbit and the blue ellipse indicates the science orbit. Planets and trajectories are not to scale. Diagrams are schematics for illustrative purposes only.

OCEANUS would be captured around Uranus via an impulsive burn into a 120-day period orbit, with an apoapsis of 200 Uranus radii $\left(\mathrm{R}_{\mathrm{U}}\right)$, approximately 2 hours following probe entry. Intersection with the $\zeta$-ring would be prevented by avoiding the approach conditions highlighted in red in Figure 2. After one orbital period, OCEANUS would pump down to a 30day science orbit with an apoapsis of $77 \mathrm{R}_{\mathrm{U}}$ and a periapsis of $1.1 \mathrm{R}_{\mathrm{U}}$, and an inclination of $69^{\circ}$. The nominal mission would be 1.5 years and 14 orbits (necessary to achieve OCEANUS' interior science objective). During this period, OCEANUS would avoid passing through the rings with the closest node-crossing transitioning between the planet and the rings from $1.32 \mathrm{R}_{\mathrm{U}}$ to $1.28 \mathrm{R}_{\mathrm{U}}$, and the furthest node-crossing transitioning outside the rings from $6.2 \mathrm{R}_{\mathrm{U}}$ to $6.75 \mathrm{R}_{\mathrm{U}}$. Proximity to the rings would be one of the major risks of the mission, because the Uranian rings are not well understood [24]. Continuous tracking would be employed when the spacecraft is less than $1.5 \mathrm{R}_{\mathrm{U}}$ from Uranus (except during occultation), to enable gravity measurements at closest approach; occultation measurements would be made during passage behind Uranus (Figure 3). An approach B-plane angle of $\sim 50^{\circ}$ results in minimizing line-of-sight blockage to support gravity science with a maximum of $40 \%$ time spent in occultation when the spacecraft is less than $1.5 \mathrm{R}_{\mathrm{U}}$ from the planet (Figure 2). Occultation will not overlap periapsis passage during any orbit. Observations with the magnetometer could be conducted at any time in the orbit, but magnetopause and bow shock crossings are of particular interest (Figure 3). Following any extended missions, the spacecraft would deorbit and impact Uranus. 


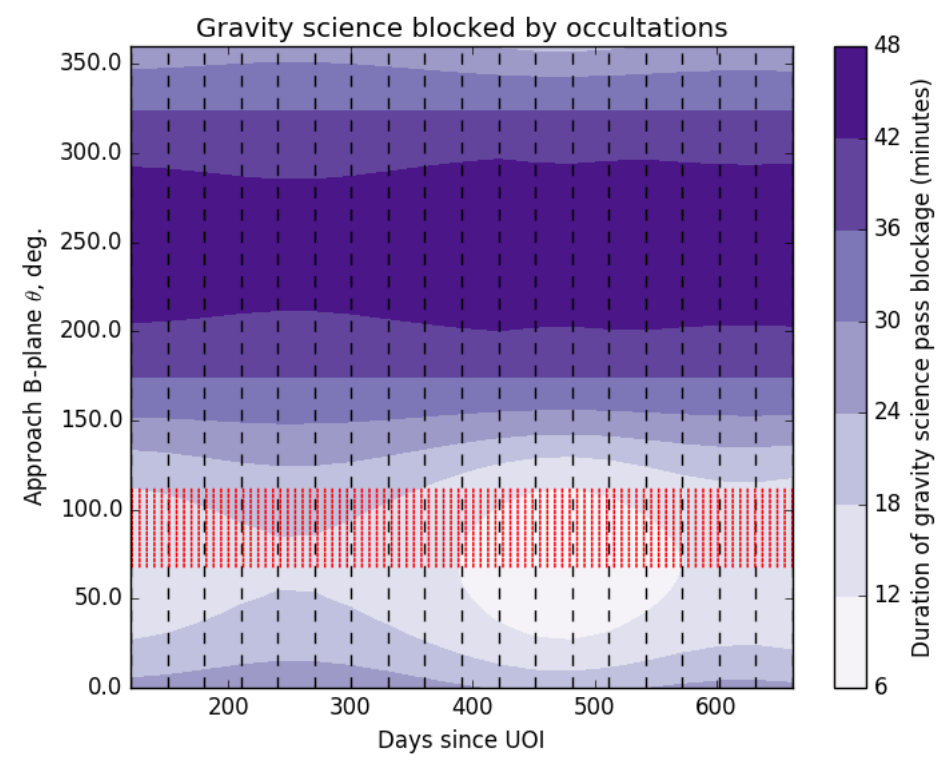

Figure 2: Gravity science blocked by occultation as a function of approach B-plane angle and days since Uranus orbit insertion. The plot starts when OCEANUS enters its science orbit (after the 120-day capture orbit) with vertical dashed lines indicating the times at which OCEANUS would be at periapsis. An approach B-plane angle in the range indicated in red would result in intersection with the $\zeta$-ring and would be avoided.

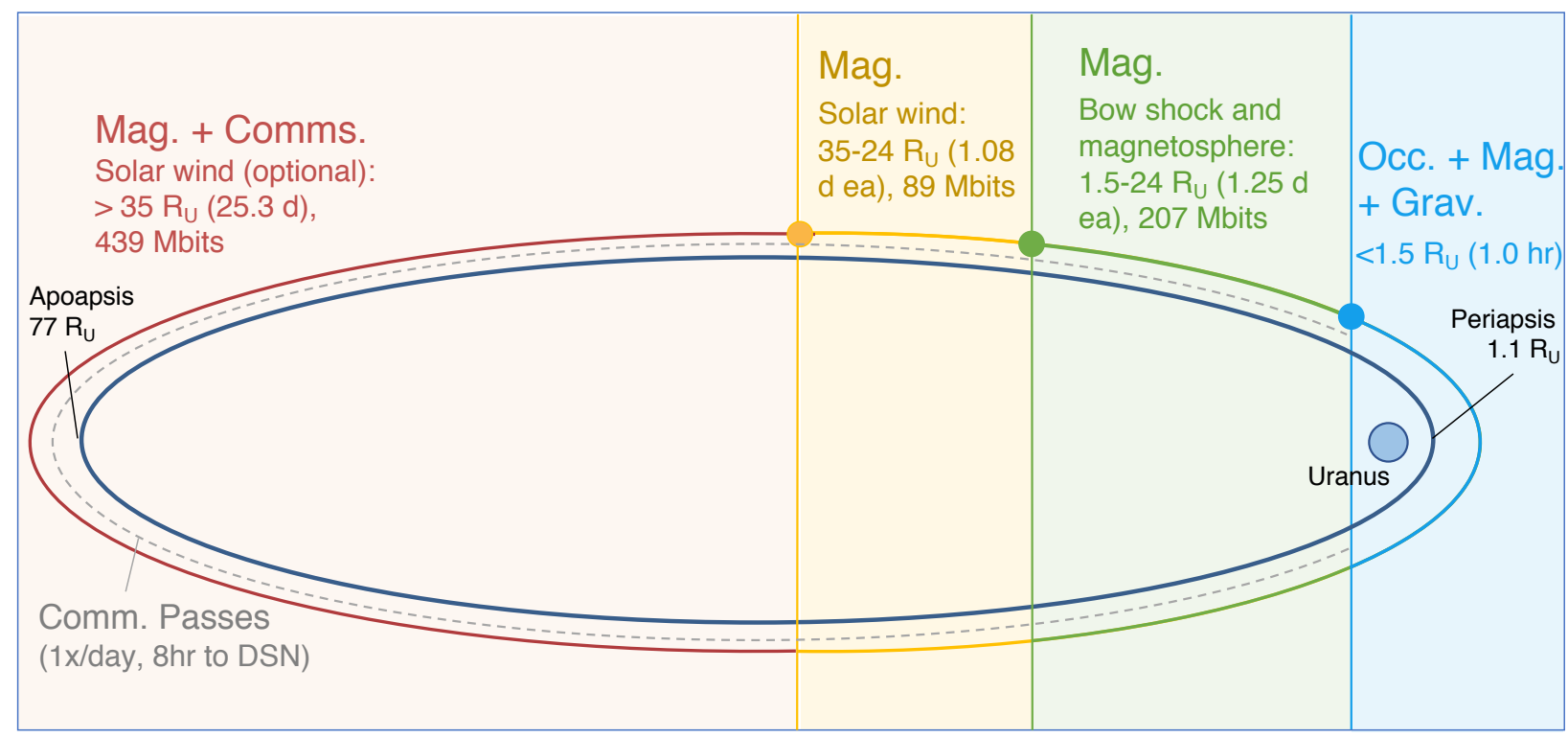

Figure 3: OCEANUS' 30-day science orbit with periapsis at $1.1 R U$ and apoapsis at $77 R U$ including: continuous tracking for gravity measurements ("Grav.") when $r<1.5$ Ru except during occultation (see section 3); magnetometer observations ("Mag.") at any time including magnetopause and bow shock crossings; radio occultation observations ("Occ.") with a maximum of $40 \%$ overlap with gravity measurements (see Figure 2); and communication of data back to Earth ("Comms."). The duration of each science phase is indicated in parentheses. Durations labeled 'ea' (each) indicate that the measurements would be conducted for that duration both before and after periapsis passage. Planning was modeled with $\mathrm{J}_{2}$ perturbations even though Uranus is displayed as a point-mass in the above schematic for illustrative purposes.

\section{Science Payload}


OCEANUS' science objectives could be achieved with only a magnetometer system and utilization of the radio system for science. Additionally, the deployment of an optional atmospheric probe could further enhance the science return of the mission, allowing additional decadal science objectives to be achieved. Estimates of cost, mass, and power for these instruments were based on the heritage instruments mentioned in the following sections.

\subsection{Magnetometers}

The mounting of magnetometers on the spacecraft would play a crucial role in understanding the peculiarities in the tilt of the Uranian magnetosphere and the origin of the planetary magnetic field. By measuring the strength and direction of the magnetic field at several distances from the planet and at different magnetic latitudes, magnetic longitudes, and points in time (Figure 3), OCEANUS' magnetometers would help constrain models for dynamo generation and characterize the three-dimensional planetary magnetic field structure (e.g. bow shock and magnetopause distance upstream from planet) at different solar wind conditions. These observations would help constrain MHD models of the magnetospheric system [25-27] and, combined with plasma and wave information from the Voyager flyby [28-31], lead to significant improvement in our understanding of the solar wind-magnetosphere interaction at Uranus. Information about the magnetospheric current systems would be deduced from magnetic field structures, as has been done at Earth [32,33].

The spacecraft would include a dual-technique magnetometer composed of a Fluxgate Magnetometer (FGM), and a Helium magnetometer capable of operating in a scalar mode (SHM) or a vector mode (VHM). The magnetometers are both mounted on an $11 \mathrm{~m}$ boom, away from contamination caused by any magnetic fields arising from the spacecraft itself (Figure 4). The science objectives require high-sensitivity measurements taken over a wide range of magnetic fields. The FGM, mounted at the midpoint of the boom at $5.5 \mathrm{~m}$, allows for the measurement of large dynamic ranges between $+/-44,000 \mathrm{nT}$. However, achieving an accurate measurement of a weak field component in a strong field with only an FGM requires precise attitude knowledge [34]. This can be solved by including an S/VHM, mounted at the end of the boom. In scalar mode, the Helium magnetometer (SHM) measures the field magnitude directly, enabling precise absolute calibration of the FGM. The SHM is able to measure weak field components in a strong background field with an accuracy of 1 part in $10^{5}$. Therefore, with the dual-technique magnetometer system, OCEANUS would be able to determine the internal magnetic field of Uranus to high orders with high precision, and the fields in the local environment to unprecedented sensitivities. A dual-technique magnetometer system such as this one was employed by the Cassini Mission under the "Cassini Magnetic Field Investigation" [34].

\subsection{Radio Science System}

\subsubsection{Gravity}

An orbiter can conduct a more detailed study of a planet's gravity field than is possible with a flyby mission such as Voyager by carefully tracking perturbations to the spacecraft's orbit through its closest approach during multiple orbits around the planet. This method is also more precise than is possible from remote observations of the orbits of natural satellites. Although [35] were able to better constrain the gravitational harmonics of Uranus than those of Neptune [36] by tracking the motions of the Uranian satellites, they were not able to constrain $\mathrm{J}_{6}$. Our combination of orbital periapsis distance and number of orbits was specifically designed to allow 
us to constrain the spherical harmonic solution to degree and order 6 . This would be achieved by tracking the Doppler shift in radio frequencies transmitted by the spacecraft. As such, OCEANUS would conduct synchronous $\mathrm{X}$ and Ka band one-way transmission during periapsis entrance and exit with continuous tracking at distances less than $1.5 \mathrm{R}_{\mathrm{U}}$ from Uranus (except during occultation; see section 3 and Figure 2), with the nominal mission including 14 orbits. This technique is similar to that employed by Cassini and Juno [37,38].

\subsubsection{Occultations}

The radio science system would also be employed for atmospheric studies by transmitting through Uranus' atmosphere during occultations (a method first used by [39] to study the Martian atmosphere). This would enable measurements similar to those made by Voyager 2 including retrieval of the thermal structure of Uranus' atmosphere and the abundance profile of methane in the pressure range from about 0.3 mbar to 2.3 bars [40]. A comparison of OCEANUS observations (equinox) to Voyager 2 observations (southern solstice) would offer important constraints on the seasonal variability of Uranus' atmosphere.

\subsection{In Situ Probe}

While remote observations can greatly increase our understanding of a planet, noble gas abundances and isotopic ratios, which are key for understanding planetary formation and the solar nebula, can only be measured in situ $[41,42]$. OCEANUS' probe would make the first in situ measurements of: the temperature, pressure, and vertical wind profiles in Uranus' atmosphere; the location, density and composition of clouds as a function of depth; and the abundances of noble gases $\mathrm{He}, \mathrm{Ne}, \mathrm{Ar}, \mathrm{Kr}$, and $\mathrm{Xe}$ and the isotopic ratios of $\mathrm{H}, \mathrm{C}, \mathrm{N}$, and $\mathrm{O}$. OCEANUS' in situ probe would achieve these measurements using a mass spectrometer, an ultra-stable oscillator, and an atmospheric structure instrument that includes two temperature sensors, three pressure sensors, and four accelerometers. These instruments are similar to those on the Galileo probe [43-45]. The probe is expected to survive until a pressure of approximately 5 bars and return data until it passes through the methane layer which is expected to occur somewhere between 1 and 3 bars (see section 3 ).

We acknowledge that the probe would only make a single-point measurement in Uranus' atmosphere. However, it would penetrate significantly deeper than the homopause (at approximately $20 \mu \mathrm{bar}$ ); below this level, strong vertical mixing dominates over molecular diffusion [46], so measurements of non-condensable gases, like noble gases, would be representative of bulk Uranus. The measurements of bulk noble gas abundances and isotopic ratios would provide a critical constraint on planetary formation and evolution models and can only be measured with an in situ probe $[41,42]$.

\subsection{Education and Public Outreach Camera}

Although OCEANUS would not require a camera to accomplish its science objectives, the mission concept includes a relatively low-cost camera similar to Juno's JunoCam [47] for education and public outreach (EPO) purposes. The camera would be a visible light, color camera with a $58^{\circ}$ field of view, an $11 \mathrm{~mm}$ focal length, and an approximately $50 \mathrm{~km} / \mathrm{pixel}$ resolution of Uranus at $3 \mathrm{R}_{\mathrm{U}}$. This offers a significantly higher resolution than the Hubble Space Telescope ( $\sim 560 \mathrm{~km} /$ pixel with the Wide Field Camera 3 [48]) and a resolution comparable to most Voyager images $[49,50]$. If power and data transmission limitations allow, the camera could also be used to provide context for scientific measurements. 


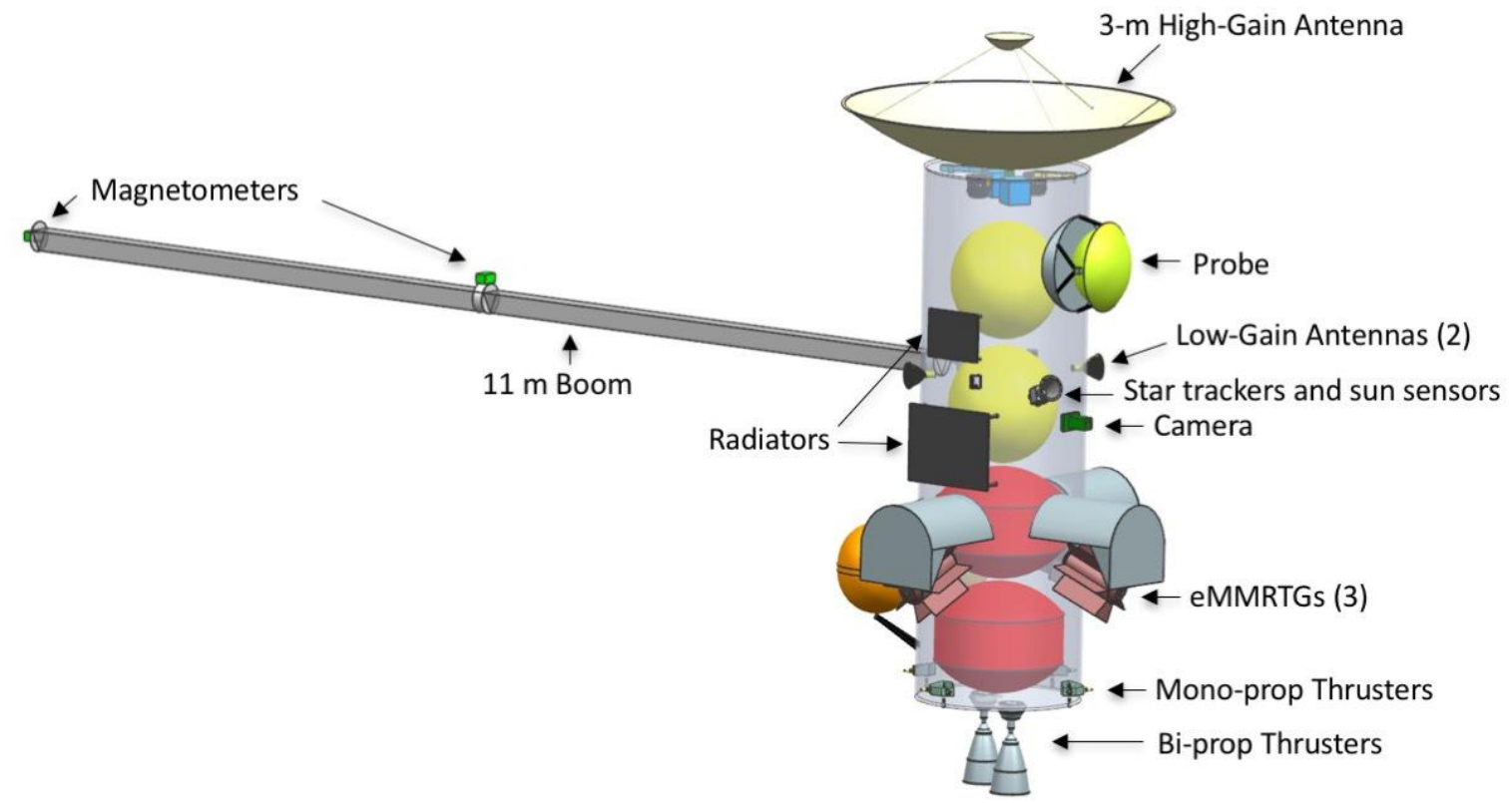

Figure 4: Spacecraft baseline configuration upon Uranus approach, after jettisoning the SEP stage and before probe deployment.

\section{Design Considerations}

Missions to ice giants are challenging due to their distance from the Sun and the Earth. Such distance limits the effectiveness of solar arrays while increasing decay of radioisotope power systems due to long cruise times and requires engineering redundancy to combat long flight times further increasing costs. Here we discuss how these considerations affected the baseline design of OCEANUS.

\subsection{Spacecraft overview}

OCEANUS would use a standard cylindrical bus, $9 \mathrm{~m}$ in height and $3 \mathrm{~m}$ in diameter (Figure 4) which could fit in an Atlas V 551 long fairing. The bus would contain four tanks, two for fuel and two for the oxidizer. In addition to the instruments discussed in the previous section, a fixed high-gain antenna, two low-gain antennas, and three enhanced MMRTGs (eMMRTGs) would be mounted to the outside of the bus. The baseline spacecraft would have a dry mass of $1533 \mathrm{~kg}$, which includes the JPL Design Principles Margin of 30\%, and a launch mass of $3939 \mathrm{~kg}$.

\subsection{Power and Thermal}

OCEANUS would be powered by three eMMRTGs. The eMMRTG is a potential upgrade of the MMRTG that would make low-risk enhancements to the previously flown MMRTG design and thus increase the end-of-mission power by more than 50\% [51]. Combined, they would provide OCEANUS with a total of approximately $290 \mathrm{~W}$ at the end of design life, which is sufficient to meet JPL's standard design principles for power contingencies. OCEANUS would use an active thermal control system with two-phase pump heating to utilize eMMRTG waste heat while only requiring $5 \mathrm{~W}$ instead of $25 \mathrm{~W}$ required by a traditional single-phase pump system. Additionally, a 32-Volt Li-ion battery with a capacity of 46 Amp-hours would be utilized to increase OCEANUS' power capability to support some operational modes. To minimize power consumption, OCEANUS would store data during closest approach to Uranus (most important for science operations) and downlink only when the spacecraft is beyond $35 \mathrm{R}_{\mathrm{U}}$ 
from the planet. However, telecom would be utilized during closest approach for gravity science and occultation measurements.

\subsection{Cost}

To estimate the cost of OCEANUS, we used a quasi-grassroots approach using JPL's Institutional Cost Model which is based on historical missions. OCEANUS has a small baseline instrument suite that would address many of the Decadal Survey objectives for a Flagship mission to Uranus for a cost of $\$ 1.18$ billion (FY2015) ${ }^{2}$. The SEP stage would cost $\$ 142.8 \mathrm{M}$, over $10 \%$ of OCEANUS' total cost, and the eMMRTGs would cost $\$ 213.2 \mathrm{M}$, approximately $20 \%$ of OCEANUS' total cost. However, OCEANUS would be able to achieve the focused set of science objectives described above using a low-cost instrument suite: $\$ 14.1 \mathrm{M}$ for the magnetometer and $\$ 8.0 \mathrm{M}$ for the EPO camera. For the purposes of the educational exercise, we assumed that the probe was contributed as furnished equipment (but did affect the total mission cost indirectly through spacecraft mass and architecture design considerations). In reality, this would be an opportunity for international collaboration. Including an atmospheric probe would allow the mission to accomplish additional Decadal Survey objectives. However, the probe could be descoped, and OCEANUS would still achieve multiple Decadal Survey objectives (Table 1), including one of the two highest-priority objectives.

\subsection{Key Trades}

\subsubsection{Flyby vs. Orbiter}

We considered designing a flyby mission concept rather than entering into orbit around Uranus; a flyby could be done without relying on SEP. This would potentially lower the total cost of the mission or allow for the addition of either a science camera or infrared radiometer onboard OCEANUS (both of which were not included due to cost-constraints). However, being in orbit is required to achieve our magnetosphere objectives and would enable study of the interior structure of Uranus using established radio science tracking methods. On a flyby mission, a Doppler seismometer could be added to the instrument payload to study the interior structure of Uranus by observing the full disk of the planet on approach and measuring acoustic modes to infer the locations of boundaries between layers in the interior. However, a Doppler seismometer has not yet been used for planetary observations, and Uranus' interior may not be active enough to produce detectable acoustic modes. We concluded that an orbiter is required not only to achieve our highest-priority science objectives relating to Uranus' magnetosphere, but also to reduce the risk and instrument cost required to study Uranus' interior.

\subsubsection{Instrument Payload}

We chose a magnetometer as the main instrument on OCEANUS, because not only does it address one of the highest priority Decadal Survey objectives for Uranus, but it is also a relatively inexpensive instrument with a cost of $\$ 14.1 \mathrm{M}$; minimizing cost was the biggest challenge in formulating OCEANUS. The magnetometer also has a relatively low mass and requires a relatively small amount of power; minimizing mass and power were also formulation challenges. We identified several other instruments, which would address Decadal Survey objectives and should be considered for a Uranus orbiter with a higher cost-cap: a high resolution

${ }^{2}$ The cost information contained in this document is of a budgetary and planning nature and is intended for informational purposes only. It does not constitute a commitment on the part of JPL and/or Caltech. 
multi-band camera, a mid-IR spectrometer, a microwave radiometer, a charged particle detector, and a plasma wave instrument. In the following paragraphs, we describe the science investigations that could be achieved with these instruments and include a brief description of their specifications and approximate cost.

If possible, we would have included a camera similar to New Horizons' LORRI [52] with a $4.6^{\circ}$ aperture, a $2.63 \mathrm{~m}$ focal length, and three $1024 \times 1024$ CCDs. Compared to OCEANUS' EPO camera, this camera would have a mass $5.1 \mathrm{~kg}$ higher, require an additional $15 \mathrm{~W}$ of power, and have a cost approximately $\$ 20 \mathrm{M}$ higher. Repeated observations with such a camera would enable tracking of cloud motion which could be used to derive wind speed profiles and directions, achieving part of the highest priority Decadal Survey objective (see Appendix for a list of Decadal Survey objectives). A camera could also be used to study the rings and satellites of Uranus, but OCEANUS' primary science phase orbit does not include any close flybys of the satellites; designing an orbit with close flybys of multiple satellites is significantly more challenging than designing an orbit that does not change during the science phase. These challenges and the budget, mass, power, and data transmission capabilities were the main reasons we opted for a less sophisticated camera. If high resolution observations of satellites were possible, key measurements would include surface images and limb observations. Surface images would be used for: crater counting to determine relative ages of the surfaces of the moons, geologic mapping to understand the overall geologic histories of the satellites, and analysis of geomorphology to understand surface evolution and to search for possible evidence of surface/subsurface material exchange. Limb observations would be used for developing shape models to understand the internal structure of satellites [53]. A camera could also be used to: search for evidence of plumes from the satellites, especially Mab, which has been suggested as a possible source of the $\mu$-ring [54]; look for evidence of precession of the rings caused by the distribution of mass in Uranus' interior (as [55] did at Saturn); and search for shepherd moons which might be maintaining the narrow distribution of Uranus rings [56]. These measurements would partially address Decadal Survey Objectives 6 and 8, which aim to improve our understanding of the Uranian satellites and rings.

Voyager measurements suggested that Uranus' heat flux is indistinguishable from zero [57], but this contradicts planetary evolution models [58]. However, the Voyager measurements had large uncertainties and only measured the thermal emission during the single Voyager flyby, whereas Uranus' thermal emission could vary with seasonal changes in atmospheric activity. Thus, a mid-IR spectrometer or radiometer is a high priority for any Uranus mission to further constrain Uranus' internal heat flux. A spectrometer would also enable the measurement of the vertical distribution and abundances of gases and complex organic molecules including the number, size distribution, composition, and optical depth profiles of clouds in the upper atmosphere $[59,60]$. For a mission that included close flybys of satellites, a mid-IR spectrometer (or radiometer) could also be used to measure the thermal inertia of the satellite surfaces [61]. These measurements could be achieved using a mid-IR spectrometer with a range of 5.8-50 $\mu \mathrm{m}$ and a spectral sampling of $10 \mathrm{~cm}^{-1}$ and $5 \mathrm{~cm}^{-1}$. Additional sub-systems include a thermal bolometer with a range of 1.5-150 $\mu \mathrm{m}$ and a solar reflectance sensor to measure the albedo in the 0.3-2.9 $\mu \mathrm{m}$ range. A mid-IR spectrometer would have a mass of $14.4 \mathrm{~kg}$, require $14.5 \mathrm{~W}$, and cost approximately $\$ 30 \mathrm{M}$. Measurements with such an instrument would address or partially address Decadal Survey objectives 1, 5, 6, 8, and 9.

Occultations, atmospheric probes, and mid-IR spectrometers cannot probe more than at most a couple tens of bars into Uranus' atmosphere [e.g. 40,44,60], but a microwave radiometer, 
with frequencies selected based on a radiative transfer model of Uranus' atmosphere, would be sensitive down to approximately 100 bars or more, and inclusion of such an instrument would enable measurements of the vertical distribution and abundances of gases down to this level (this approach is currently being used by the Juno mission [62]). A microwave radiometer could also be used to measure the microwave flux and map the atmospheric temperature structure at these lower levels, providing an important constraint on radiative transfer models [62]. These measurements could be achieved with a microwave radiometer similar to that on Juno [62] with frequency channels optimized for observing Uranus: $22 \mathrm{GHz}, 10 \mathrm{GHz}, 5.3 \mathrm{GHz}, 1.7 \mathrm{GHz}, 0.8$ GHz. It would have a mass of $46 \mathrm{~kg}$, require $32 \mathrm{~W}$ of power, and cost approximately $\$ 50 \mathrm{M}$. It would address or partially address Decadal Survey objectives 1 and 9.

Although OCEANUS' magnetometer would greatly improve our understanding of Uranus' magnetosphere, including a charged particle detector and a wave instrument in addition to a magnetometer would further constrain the physics and dynamics of the magnetosphere. The cost of these instruments would be approximately $\$ 10 \mathrm{M}$. A charged particle and plasma detector measures the energy spectra and pitch angle distributions of ions and electrons. Making these measurements at different radial distances, magnetic local times, and latitudes further constrains the structure of a planet's magnetosphere including the location of the bow shock and magnetopause [e.g. 25]. These instruments would also be capable of detecting high speed jets of ions and electrons resulting from magnetic reconnection. Measurements of the solar wind plasma outside of the magnetopause could be compared to measurements from missions to other parts of the solar system to understand how the solar wind changes with distance from the sun [63]. These measurements could be achieved with a charged particle detector similar to MESSENGER's Energetic Particle and Plasma Spectrometer [64] which was sensitive to highenergy protons with energies from $15 \mathrm{keV}$ to $3 \mathrm{MeV}$, high-energy electrons with energies from $15 \mathrm{keV}$ to $1.0 \mathrm{MeV}$, and low-energy ions with energies from $50 \mathrm{eV} / \mathrm{charge}$ to $20 \mathrm{keV} /$ charge. The instrument has a mass of $10.3 \mathrm{~kg}$ and requires $13.7 \mathrm{~W}$ of power. A plasma wave instrument would measure the electric field at different radial distances, magnetic local times, and latitudes, and would help constrain magnetospheric convection and the location of the magnetospheric cusp. It could also be used to measure the magnetotail twisting rate. These measurements could be made with a plasma wave instrument sensitive to frequencies ranging from $50 \mathrm{~Hz}$ to $40 \mathrm{MHz}$ in the electric field and $50 \mathrm{~Hz}$ to $20 \mathrm{KHz}$ in the magnetic field. Such an instrument would have a mass of $5 \mathrm{~kg}$ and require $4 \mathrm{~W}$ of power. Including a charged particle detector and a plasma wave instrument in addition to magnetometers would enable OCEANUS to more thoroughly address Decadal Survey Objectives 2 and 7.

\section{Conclusion}

By addressing a focused set of science objectives, OCEANUS would achieve high science return with a small suite of heritage instruments. With only a magnetometer, a radio science system, and a $\$ 1.18$ billion budget, OCEANUS can achieve multiple objectives outlined by the Decadal Survey for a Flagship mission to Uranus. Due to the relatively low cost of the magnetometer system and the utilization of the radio system for science, this is currently the lowest cost instrument suite possible that would address multiple decadal survey objectives at Uranus. This instrument suite also has a relatively low mass and power consumption, which further enhances its value for use on a New-Frontiers-class mission. A contributed in situ atmospheric probe would enable OCEANUS to achieve additional priority science described by the Decadal Survey. In the event that a Flagship mission is not an option for any space agency 
during this decade, a more focused mission concept should be considered to achieve a subset of the NASA and ESA science objectives at Uranus.

\section{Acknowledgements}

We thank the organizers of the Planetary Science Summer School (especially Charles Budney, Karl Mitchell, Leslie Lowes, Laurita Borst and Jessica Parker), the JPL Team X mentors who assisted us in this study, William Smythe whose feedback improved the manuscript, and Elizabeth Turtle and one anonymous reviewer who provided thorough reviews and constructive suggestions which greatly improved the paper. We also thank the NASA Science Mission Directorate Planetary Science Division for providing the funding for the Planetary Science Summer School. This research was carried out at the Jet Propulsion Laboratory, California Institute of Technology, under a contract with the National Aeronautics and Space Administration. The information provided about the Oceanus mission concept is pre-decisional and is presented for planning and discussion purposes only.

\section{References}

[1] J.J. Fortney, N. Nettelmann, The interior structure, composition, and evolution of giant planets, Space Sci. Rev. 152 (2010) 423-447. doi:10.1007/s11214-009-9582-x.

[2] D.J. Stevenson, Planetary magnetic fields, Earth Planet. Sci. Lett. (2003) 1-11. doi:10.1029/GL006i003p00213.

[3] A. Izidoro, A. Morbidelli, S.N. Raymond, F. Hersant, A. Pierens, Accretion of Uranus and Neptune from inward-migrating planetary embryos blocked by Jupiter and Saturn, Astron. Astrophys. 582 (2015) A99. doi:10.1051/0004-6361/201425525.

[4] A. Morbidelli, S.N. Raymond, Special Section :, J. Geophys. Res. Planets. 121 (2016) 1962-1980. doi:10.1002/2016JE005088.Over.

[5] P.R. Mahaffy, H.B. Niemann, A. Alpert, S.K. Atreya, J. Demick, T.M. Donahue, D.N. Harpold, T.C. Owen, Noble gas abundance and isotope ratios in the atmosphere of Jupiter from the Galileo Probe Mass Spectrometer, J. Geophys. Res. 105 (2000) 15061-15071. doi:10.1029/1999JE001224.

[6] NASA Exoplanet Archive, (n.d.). http://exoplanetarchive.ipac.caltech.edu/docs/counts_detail.html (accessed January 27, 2017).

[7] L.A. Rogers, Most 1.6 Earth-radius planets are not rocky, Astrophys. J. 801 (2015) 41. doi:10.1088/0004-637X/801/1/41.

[8] H.B. Hammel, The Ice Giant Systems of Uranus and Neptune, in: Sol. Syst. Updat., Springer, 2006: pp. 251-265.

[9] N. National Research Council, Vision and Voyages for Planetary Science in the Decade 2013-2022, The National Acadmies Press, Washington, DC, 2011. doi:10.17226/13117.

[10] C.J. Budney, L.L. Lowes, K.L. Mitchell, A.M. Sohus, A.S. Wessen, NASA Planetary Science Summer School: Training the Next Generation of Planetary Mission Leaders, in: 45th Lunar Planet. Sci. Conf., 2014: p. 1563. doi:10.1002/jgre.20118.

[11] C.S. Arridge, The Science Case for an Orbital Mision to Uranus: Exploring the Origins and Evolution of Ice Giant Planets, in: Cosm. Vis. White Pap. Submitt. Response to ESA's Call Sci. Themes L2 L3 Mission., 2013. http://sci.esa.int/sciencee/www/object/doc.cfm?fobjec tid=52029.

[12] C.S. Arridge, N. Achilleos, J. Agarwal, C.B. Agnor, R. Ambrosi, N. André, S. V. 
Badman, K. Baines, D. Banfield, M. Barthélémy, M.M. Bisi, J. Blum, T. BocanegraBahamon, B. Bonfond, C. Bracken, P. Brandt, C. Briand, C. Briois, S. Brooks, J. CastilloRogez, T. Cavalié, B. Christophe, A.J. Coates, G. Collinson, J.F. Cooper, M. Costa-Sitja, R. Courtin, I.A. Daglis, I. De Pater, M. Desai, D. Dirkx, M.K. Dougherty, R.W. Ebert, G. Filacchione, L.N. Fletcher, J. Fortney, I. Gerth, D. Grassi, D. Grodent, E. Grün, J. Gustin, M. Hedman, R. Helled, P. Henri, S. Hess, J.K. Hillier, M.H. Hofstadter, R. Holme, M. Horanyi, G. Hospodarsky, S. Hsu, P. Irwin, C.M. Jackman, O. Karatekin, S. Kempf, E. Khalisi, K. Konstantinidis, H. Krüger, W.S. Kurth, C. Labrianidis, V. Lainey, L.L. Lamy, M. Laneuville, D. Lucchesi, A. Luntzer, J. MacArthur, A. Maier, A. Masters, S.

McKenna-Lawlor, H. Melin, A. Milillo, G. Moragas-Klostermeyer, A. Morschhauser, J.I. Moses, O. Mousis, N. Nettelmann, F.M. Neubauer, T. Nordheim, B. Noyelles, G.S. Orton, M. Owens, R. Peron, C. Plainaki, F. Postberg, N. Rambaux, K. Retherford, S. Reynaud, E. Roussos, C.T. Russell, A.M. Rymer, R. Sallantin, A. Sánchez-Lavega, O. Santolik, J. Saur, K.M. Sayanagi, P. Schenk, J. Schubert, N. Sergis, E.C. Sittler, A. Smith, F. Spahn, R. Srama, T. Stallard, V. Sterken, Z. Sternovsky, M. Tiscareno, G. Tobie, F. Tosi, M. Trieloff, D. Turrini, E.P. Turtle, S. Vinatier, R. Wilson, P. Zarka, The science case for an orbital mission to Uranus: Exploring the origins and evolution of ice giant planets, Planet. Space Sci. 104 (2014) 122-140. doi:10.1016/j.pss.2014.08.009.

[13] M.D. Hofstadter, A. Simon, K. Reh, J. Elliot, Ice Giants Pre-Decadal Study Final Report, 2017. https://www.lpi.usra.edu/icegiants/mission_study/.

[14] N. National Research Council, Solar and Space Physics: A Science for a Technological Society, National Academies Press, Washington, DC, 2013. doi:10.17226/13060.

[15] S.D. Wall, Use of Concurrent Engineering in Space Mission Design, Proc. EuSEC. (2000).

[16] S.D. Wall, Model-based engineering design for space missions, Aerosp. Conf. 2004. Proceedings. 2004 IEEE. 6 (2004) 3907-3915.

[17] L.A. Sromovsky, P.M. Fry, J.H. Kim, Methane on Uranus: The case for a compact CH4 cloud layer at low latitudes and a severe $\mathrm{CH} 4$ depletion at high-latitudes based on reanalysis of Voyager occultation measurements and STIS spectroscopy, Icarus. 215 (2011) 292-312. doi:10.1016/j.icarus.2011.06.024.

[18] E. Karkoschka, M.G. Tomasko, The haze and methane distributions on Uranus from HSTSTIS specroscopy, Icarus. 202 (2009) 287-309. doi:10.1016/j.icarus.2009.02.010.

[19] L.A. Sromovsky, E. Karkoschka, P.M. Fry, H.B. Hammel, I. de Pater, K. Rages, Methane depletion in both polar regions of Uranus inferred from HST/STIS and Keck/NIRC2 observations, Icarus. 238 (2014) 137-155. doi:10.1016/j.icarus.2014.05.016.

[20] K. de Kleer, S. Luszcz-Cook, I. de Pater, M. Ádámkovics, H.B. Hammel, Clouds and aerosols on Uranus: Radiative transfer modeling of spatially-resolved near-infrared Keck spectra, Icarus. 256 (2015) 120-137. doi:10.1016/j.icarus.2015.04.021.

[21] L.A. D’Amario, L.E. Bright, A.A. Wolf, Galileo trajectory design, Space Sci. Rev. 60 (1992) 23-78. doi:10.1007/BF00216849.

[22] R.E. Young, The Galileo probe mission to Jupiter: Science overview, J. Geophys. Res. 103 (1998) 22775-22790. doi:10.1029/98JE01051.

[23] R.J. Haw, P.G. Antreasian, T.P. McElrath, E.J. Graat, F.T. Nicholson, Navigating Galileo at Jupiter Arrival, J. Spacecr. Rockets. 34 (1997) 1-6. doi:10.2514/2.3240.

[24] I. de Pater, S.G. Gibbard, H.B. Hammel, Evolution of the dusty rings of Uranus, Icarus. 180 (2006) 186-200. doi:10.1016/j.icarus.2005.08.011. 
[25] G.-H. Voigt, K.W. Behannon, N.F. Ness, Magnetic field and current structures in the magnetosphere of Uranus, J. Geophys. Res. 92 (1987) 15337-15346.

doi:10.1029/JA092iA13p15337.

[26] G. Tóth, D. Kovács, K.C. Hansen, T.I. Gombosi, Three-dimensional MHD simulations of the magnetosphere of Uranus, J. Geophys. Res. Sp. Phys. 109 (2004) 1-17. doi:10.1029/2004JA010406.

[27] A. Masters, Magnetic reconnection at Uranus' magnetopause, J. Geophys. Res. Sp. Phys. 119 (2014) 5520-5538. doi:10.1002/2014JA020077.

[28] H.S. Bridge, J.W. Belcher, B. Coppi, A.J. Lazarus, R.L. McNutt, S. Olbert, J.D. Richardson, M.R. Sands, R.S. Selesnick, J.D. Sullivan, R.E. Hartle, K.W. Ogilvie, E.C. Sittler, F. Bagenal, R.S. Wolff, V.M. Vasyliunas, G.L. Siscoe, C.K. Goertz, A. Eviatar, Plasma observations near Uranus: initial results from Voyager 2., Science. 233 (1986) 8993. doi:10.1126/science.246.4936.1478.

[29] B.H. Mauk, S.M. Krimigis, E.P. Keath, A.F. Cheng, T.P. Armstrong, L.J. Lanzerotti, G. Gloeckler, D.C. Hamilton, The hot plasma and radiation environment of the Uranian magnetosphere, J. Geophys. Res. 92 (1987) 15283-15308. doi:10.1029/JA092iA13p15283.

[30] D.A. Gurnett, W.S. Kurth, F.L. Scarf, R.L. Poynter, First plasma wave observations at Uranus, Science. 233 (1986) 106-109. doi:10.1126/science.233.4759.106.

[31] W.S. Kurth, D.D. Barbosa, D.A. Gurnett, F.L. Scarf, Electrostatic waves in the magnetosphere of Uranus, J. Geophys. Res. 92 (1987) 15225-15233. doi:10.1029/JA092iA13p15225.

[32] G.D. Mead, Deformation of the geomagnetic field by the solar wind, J. Geophys. Res. 69 (1964) 1181-1195. doi:10.1029/JZ069i007p01181.

[33] J.W. Gjerloev, S. Ohtani, T. Iijima, B. Anderson, J. Slavin, G. Le, Characteristics of the terrestrial field-aligned current system, Ann. Geophys. 29 (2011) 1713-1729. doi:10.5194/angeo-29-1713-2011.

[34] M.K. Dougherty, S. Kellock, D.J. Southwood, A. Balogh, E.J. Smith, B.T. Tsurutani, B. Gerlach, K.H. Glassmeier, F. Gleim, C.T. Russell, G. Erdos, The Cassini magnetic field investigation, in: Cassini-Huygens Mission, Springer, 2004: pp. 331-383.

[35] R.A. Jacobson, The orbits of the Uranian satellites and rings, the gravity field of the Uranian system, and the orientation of the pole of Uranus, Astron. J. 148 (2014) 13. doi:10.1088/0004-6256/148/5/76.

[36] R.A. Jacobson, The orbits of the Neptunian satellites and the orientation of the pole of Neptune, Astron. J. 137 (2009) 4322-4329. doi:10.1088/0004-6256/137/5/4322.

[37] A.J. Kliore, J.D. Anderson, J.W. Armstrong, S.W. Asmar, C.L. Hamilton, N.J. Rappaport, H.D. Wahlquist, R. Ambrosini, F.M. Flasar, R.G. French, L. Iess, E.A. Marouf, A.F. Nagy, Cassini radio science, Space Sci. Rev. 115 (2005) 1-70. doi:10.1007/s11214-0041436-y.

[38] G. Tommei, L. Dimare, D. Serra, A. Milani, On the Juno radio science experiment: Models, algorithms and sensitivity analysis, Mon. Not. R. Astron. Soc. 446 (2014) 30893099. doi:10.1093/mnras/stu2328.

[39] A. Kliore, D.L. Cain, G.S. Levy, V.R. Eshleman, G. Fjeldbo, F.D. Drake, Occultation experiment: Results of the first direct measurement of Mars's atmosphere and ionosphere., Science. 149 (1965) 1243-1248. doi:10.1126/science.149.3689.1243.

[40] G.F. Lindal, J.R. Lyons, D.N. Sweetnam, V.R. Eshleman, D.P. Hinson, G.L. Tyler, The 
atmosphere of Uranus: Results of radio occultation measurements with Voyager 2, J. Geophys. Res. 92 (1987) 14987-15001. doi:10.1029/GL017i010p01733.

[41] T. Owen, Atmospheric probes: needs and prospects, in: A. Wilson (Ed.), Proc. Int. Work. Planet. Probe Atmos. Entry Descent Trajectory Anal. Sci., 2004: pp. 7-11.

[42] T. Owen, T. Encrenaz, Compositional constraints on giant planet formation, Planet. Space Sci. 54 (2006) 1188-1196. doi:10.1016/j.pss.2006.05.030.

[43] H.B. Niemann, D.N. Harpold, S.K. Atreya, G.R. Carignan, D.M. Hunten, T.C. Owen, Galileo Probe Mass Spectrometer experiment, Space Sci. Rev. 60 (1992) 111-142. doi:10.1007/BF00216852.

[44] J.B. Pollack, D.H. Atkinson, A. Seiff, J.D. Anderson, Retrieval of a wind profile from the Galileo probe telemetry signal, Space Sci. Rev. 60 (1992) 143-178. doi:10.1007/BF00216853.

[45] A. Seiff, T.C.D. Knight, The Galileo probe atmosphere structure instrument, Space Sci. Rev. 60 (1992) 203-232. doi:10.1007/BF00216855.

[46] J.I. Lunine, The atmospheres of Uranus and Neptune, Annu. Rev. Astron. Astrophys. 31 (1993) 217-263. doi:10.1146/annurev.aa.31.090193.001245.

[47] C.J. Hansen, M.A. Caplinger, A. Ingersoll, M.A. Ravine, E. Jensen, S. Bolton, G. Orton, Junocam: Juno's outreach camera, Space Sci. Rev. (2014). doi:10.1007/s11214-014-0079$\mathrm{x}$.

[48] P.G.J. Irwin, M.H. Wong, A.A. Simon, G.S. Orton, D. Toledo, HST/WFC3 observations of Uranus' 2014 storm clouds and comparison with VLT/SINFONI and IRTF/Spex observations, Icarus. 288 (2017) 99-119. doi:10.1016/j.icarus.2017.01.031.

[49] B.A. Smith, L.A. Soderblom, R. Beebe, D. Bliss, J.M. Boyce, A. Brahic, G.A. Briggs, R.H. Brown, S.A. Collins, A.F. Cook, S.K. Croft, J.N. Cuzzi, G. Danielson, M. Davies, T. Dowling, D. Godfrey, C. Hansen, C. Harris, G. Hunt, A. Ingersoll, T. Johnson, R. Krauss, H. Masursky, D. Morrison, T. Owen, J. Plescia, J. Pollack, C. Porco, K. Rages, C. Sagan, E. Shoemaker, L. Sromovsky, C. Stoker, R. Strom, V. Suomi, S. Synnott, R. Terrile, P. Thomas, W. Thompson, J. Veverka, Voyager 2 in the Uranian system: Imaging science results, Science (80-. ). 233 (1986) 43-64.

[50] K. Rages, J.B. Pollack, M.G. Tomasko, L.R. Doose, Properties of scatterers in the troposphere and lower stratosphere of Uranus based on Voyager imaging data, Icarus. 89 (1991) 359-376. doi:10.1016/0019-1035(91)90183-T.

[51] T.C. Holgate, R. Bennett, T. Hammel, T. Caillat, S. Keyser, B. Sievers, Increasing the efficiency of the multi-mission radioisotope thermoelectric generator, J. Electron. Mater. 44 (2015) 1814-1821. doi:10.1007/s11664-014-3564-9.

[52] A. Cheng, H. Weaver, S. Conard, M. Morgan, O. Barnouin-Jha, J. Boldt, K. Cooper, E. Darlington, M. Grey, J. Hayes, K. Kosakowski, T. Magee, E. Rossano, D. Sampath, C. Schlemm, H. Taylor, Long-range reconnaissance imager on New Horizons, in: C.T. Russel (Ed.), New Horizons, Springer, New York, NY, 2009: pp. 189-215.

[53] P.C. Thomas, J.A. Burns, P. Helfenstein, S. Squyres, J. Veverka, C. Porco, E.P. Turtle, A. McEwen, T. Denk, B. Giese, T. Roatsch, T. V. Johnson, R.A. Jacobson, Shapes of the saturnian icy satellites and their significance, Icarus. 190 (2007) 573-584. doi:10.1016/j.icarus.2007.03.012.

[54] I. De Pater, H.B. Hammel, S.G. Gibbard, M.R. Showalter, New dust belts of Uranus: One ring, two ring, red ring, blue ring, Science (80-. ). 312 (2006) 92-94.

doi:10.1126/science.1125110. 
[55] M.M. Hedman, P.D. Nicholson, Kronoseismology: Using density waves in Saturn's c ring to probe the planet's interior, Astron. J. 146 (2013). doi:10.1088/0004-6256/146/1/12.

[56] R. French, P. Nicholson, C. Porco, E. Marouf, Dynamics and structure of the Uranian Rings, in: Uranus, 1991: pp. 327-409.

[57] J.C. Pearl, B.J. Conrath, R.A. Hanel, J.A. Pirraglia, A. Coustenis, The albedo, effective temperature, and energy balance of Uranus, as determined from Voyager IRIS data, Icarus. (1990) 12-28. doi:10.1016/0019-1035(90)90155-3.

[58] N. Nettelmann, R. Helled, J.J. Fortney, R. Redmer, New indication for a dichotomy in the interior structure of Uranus and Neptune from the application of modified shape and rotation data, Planet. Space Sci. 77 (2013) 143-151. doi:10.1016/j.pss.2012.06.019.

[59] F.M. Flasar, V.G. Kunde, M.M. Abbas, R.K. Achterberg, P. Ade, A. Barucci, B. Bézard, G. Bjoraker, J. Brasunas, S. Calcutt, R. Carlson, C. Césarsky, B. Conrath, A. Coradini, R. Courtin, A. Coustenis, S. Edberg, S. Edgington, C. Ferrari, T. Fouchet, D. Gautier, P. Gierasch, K. Grossman, P. Irwin, D. Jennings, L. E, A. Mamoutkine, A. Marten, J. Meyer, C. Nixon, G. Orton, T. Owen, J. Pearl, R. Prangé, F. Raulin, P. Read, P. Romani, R. Samuelson, M. Segura, M. Showalter, A. Simon-Miller, M. Smith, J. Spencer, L. Spilker, F. Taylor, Exploring the Saturn system in the thermal infrared: The composite infrared spectrometer, Space Sci. Rev. 115 (2004) 169-297.

[60] L.N. Fletcher, G.S. Orton, N.A. Teanby, P.G.J. Irwin, G.L. Bjoraker, Methane and its isotopologues on Saturn from Cassini/CIRS observations, Icarus. 199 (2009) 351-367. doi:10.1016/j.icarus.2008.09.019.

[61] C.J.A. Howett, J.R. Spencer, J. Pearl, M. Segura, Thermal inertia and bolometric Bond albedo values for Mimas, Enceladus, Tethys, Dione, Rhea and Iapetus as derived from Cassini/CIRS measurements, Icarus. 206 (2010) 573-593. doi:10.1016/j.icarus.2009.07.016.

[62] M.A. Janssen, J.E. Oswald, S.T. Brown, S. Gulkis, S.M. Levin, S.J. Bolton, M.D. Allison, S.K. Atreya, D. Gautier, A.P. Ingersoll, J.I. Lunine, G.S. Orton, T.C. Owen, P.G. Steffes, V. Adumitroaie, A. Bellotti, L.A. Jewell, C. Li, L. Li, S. Misra, F.A. Oyafuso, D. SantosCosta, E. Sarkissian, R. Williamson, J.K. Arballo, A. Kitiyakara, A. Ulloa-Severino, J.C. Chen, F.W. Maiwald, A.S. Sahakian, P.J. Pingree, K.A. Lee, A.S. Mazer, R. Redick, R.E. Hodges, R.C. Hughes, G. Bedrosian, D.E. Dawson, W.A. Hatch, D.S. Russell, N.F. Chamberlain, M.S. Zawadski, B. Khayatian, B.R. Franklin, H.A. Conley, J.G. Kempenaar, M.S. Loo, E.T. Sunada, V. Vorperion, C.C. Wang, MWR: Microwave Radiometer for the Juno Mission to Jupiter, 2017. doi:10.1007/s11214-017-0349-5.

[63] A. Masters, J.A. Slavin, G.A. Dibraccio, T. Sundberg, R.M. Winslow, C.L. Johnson, B.J. Anderson, H. Korth, A comparison of magnetic overshoots at the bow shocks of Mercury and Saturn, J. Geophys. Res. Sp. Phys. 118 (2013) 4381-4390. doi:10.1002/jgra.50428.

[64] G.B. Andrews, T.H. Zurbuchen, B.H. Mauk, H. Malcom, L.A. Fisk, G. Gloeckler, G.C. Ho, J.S. Kelley, P.L. Koehn, T.W. Lefevere, S.S. Livi, R.A. Lundgren, J.M. Raines, The energetic particle and plasma spectrometer instrument on the MESSENGER spacecraft, Space Sci. Rev. 131 (2007) 523-556. doi:10.1007/s11214-007-9272-5.

\section{Appendix}

This appendix provides the science objectives for Uranus as defined by the U.S. Decadal Survey [9] and an ESA white paper [11,12]. OCEANUS would make progress towards achieving objectives listed in bold; OCEANUS would only make progress towards achieving objectives 
listed in bold italic if an atmospheric probe is included.

U.S. Decadal Survey objectives [9]:

Highest priority objectives:

1. "Determine the atmospheric zonal winds, composition, and structure at high spatial resolution, as well as the temporal evolution of atmospheric dynamics."

2. "Understand the basic structure of the planet's magnetosphere as well as the highorder structure and temporal evolution of the planet's interior dynamo."

Medium priority objectives:

3. "Determine the noble gas abundances (helium, neon, argon, krypton, and xenon) and isotopic ratios of hydrogen, carbon, nitrogen, and oxygen in the planet's atmosphere and the atmospheric structure at the probe descent location."

4. "Determine internal mass distribution."

5. "Determine the horizontal distribution of atmospheric thermal emission, as well as the upper-atmospheric thermal structure and changes with time and location at low resolution."

6. "Determine the geology, geophysics, surface composition, and interior structure of large satellites."

Lower priority objectives:

7. "Measure the magnetic field, plasma, and currents to determine how the tilted/offset/rotating magnetosphere interacts with the solar wind over time."

8. "Determine the composition, structure, particle-size distribution, dynamical stability, and evolutionary history of the rings, as well as the geology, geophysics, and surface composition of small satellites."

9. "Determine the vertical profile of zonal winds as a function of depth in the atmosphere, in addition to the presence of clouds as a function of depth in the atmosphere."

ESA white paper main science questions [11,12]:

1. Uranus as an Ice Giant Planet

1.1. What is the internal structure and composition of Uranus?

1.2. Why does Uranus emit very little heat?

1.3. What is the configuration and origin of Uranus' highly asymmetric magnetic field?

1.4. What is the rotation rate of Uranus' interior?

1.5. How is Uranus' weather structure and composition influenced by its unique seasons?

1.6. What processes shape atmospheric chemistry and cloud formation on an Ice Giant?

1.7. What processes govern upper atmospheric structure?

2. An Ice Giant Planetary System

2.1. What is the composition of the Uranian rings?

2.2. How do dense rings behave dynamically?

2.3. How do Uranus' dusty rings work?

2.4. How do the rings and inner satellites interact?

2.5. What is the origin of the rings/satellite system?

2.6. What is the composition of the Uranian moons?

2.7. What is the origin of Uranus' moons and how have they evolved? 
3. Uranus' Aeronomy, Auroarae and Highly Asymmetrical Magnetosphere

3.1. What is the overall configuration of the Uranian magnetosphere?

3.2. What are the characteristics and origins of the Uranian aurorae?

3.3. How does magnetosphere-ionosphere-Solar Wind coupling work at Ice Giants?

Vitae

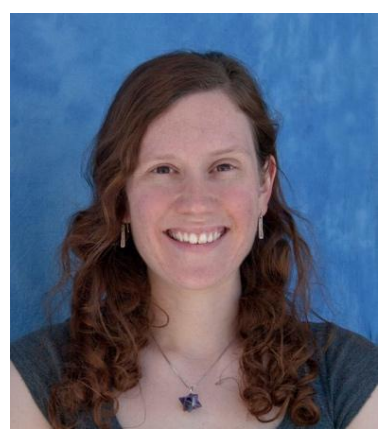

Dr. Catherine M. Elder is a postdoctoral scholar at the Jet Propulsion Laboratory, California Institute of Technology. She received her B.A. in astronomy from Cornell and her Ph.D. in Planetary Sciences from the University of Arizona. She is a member of the Lunar

Reconnaissance Orbiter's Diviner Radiometer team. Her research interests include the geology of rocky and icy bodies from their mantles through their lithospheres and crusts to their surfaces. She studies processes in these bodies through both analysis of remote sensing data and geophysical modeling.

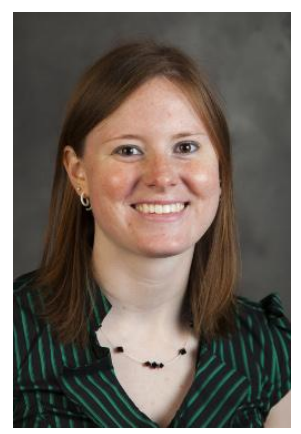

Ali M. Bramson is a PhD student at the University of Arizona's Department of Planetary Sciences/Lunar and Planetary Laboratory. She received her B.S. in Physics and AstronomyPhysics with a certificate in Computer Science from the University of Wisconsin-Madison and her M.S. in Planetary Sciences from the University of Arizona. Her research interests involve using spacecraft data (particularly images and radar) and theoretical modeling to understand planetary surface processes and the distribution of ice on the terrestrial planets and moons. 


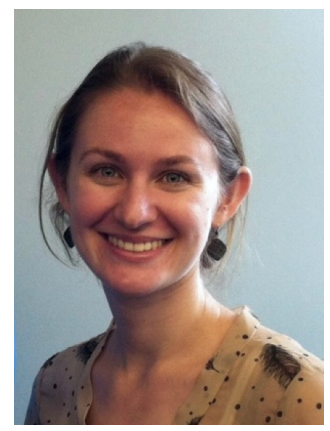

Dr. Lauren W. Blum is a research scientist at NASA Goddard Space Flight Center. She received her B.A. in physics from Dartmouth College and Ph.D. from the University of Colorado Boulder in Aerospace Engineering Sciences. Following this, she spent two years at Berkeley's Space Sciences Laboratory as an NSF postdoctoral fellow. Her research focuses on understanding the variability of Earth's radiation belts and the role of wave-particle interactions in both the acceleration and loss of energetic electrons. Her experience includes analysis of particle and field measurements from satellites, balloons, and ground stations, as well as instrumentation and small satellite design.

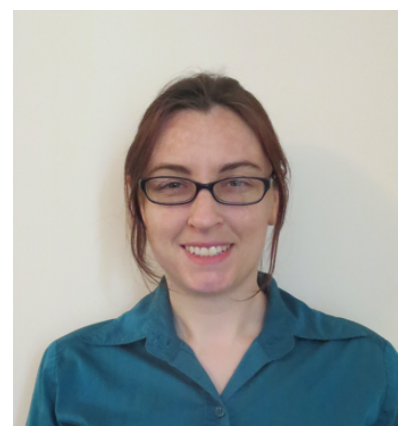

Heather T. Chilton is a PhD candidate at Georgia Institute of Technology, working with Dr. Britney Schmidt and the Dawn Science Team studying landslides on Ceres. She received her B.S. in both Geology and Physics at California State University - Fullerton. Other research includes a JPL selected CubeSAT concept study for the Europa Mission and both ridge analyses and thermodynamic modeling at Enceladus. Her research interests include using remote sensing to better understand surface geomorphologies connected with and informing us about icy and ice-rich bodies, mission studies for improved feedback between science and engineering, and science communication to the public.

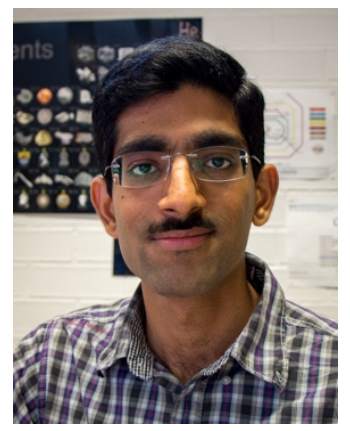

Dr. Aditya Chopra is an Astrobiology \& Planetary Science Postdoctoral Researcher at the Australian National University. During the concept study for OCEANUS in 2016, he was a 
Postdoctoral Researcher at the University of Washington. His research expertise is in the astrophysical, geochemical and biological constraints on planetary habitability. He completed his Bachelor's degree in Chemistry at the University of Western Australia. He graduated with 1st class Honours in Astronomy and a PhD in Earth Sciences at the Australian National University. $\mathrm{He}$ is investigating the role of biological regulation in maintaining a liquid water inventory on rocky planets over billions of years.

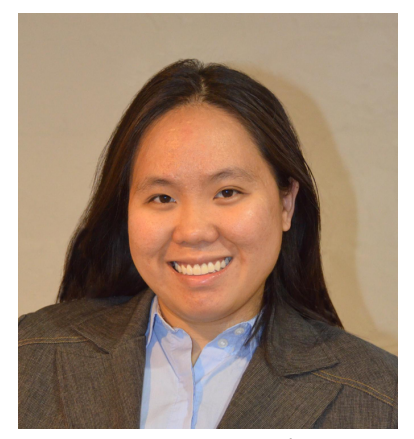

Christina Chu is a Space Physics Ph.D. candidate at the University of Alaska Fairbanks Geophysical Institute. She received her M.S. in Engineering Physics, B.S. in Engineering Physics, and B.S. in Space Physics from Embry-Riddle Aeronautical University. She received her A.S. in Mathematics and A.S. in Chemistry from Middle Georgia College. Her research interests involve using in-situ spacecraft data to understand the properties and evolution of foreshock transients.

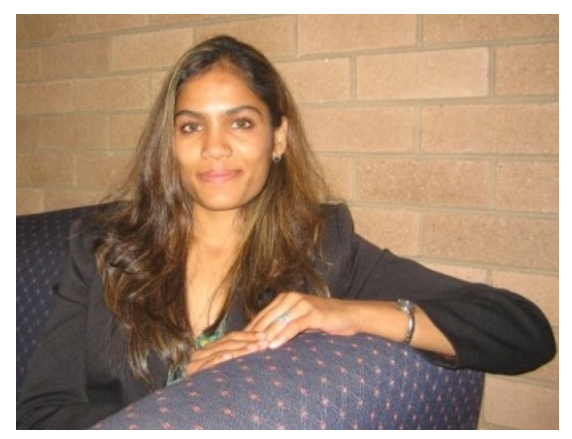

Ashwati Das is a Ph.D student at Purdue University in West Lafayette, Indiana. She received her B.S. in Aerospace Engineering from the University of Sydney in Australia, and a Masters in Aero/Astro Engineering from Purdue University. The focus of her Master's research was investigating the use of propellantless technologies for enabling space missions. She has worked with the Mars Advanced Concepts Group at NASA JPL on Sample Return studies, with NASA Marshall on mission architecture trade-studies, and with NASA Goddard on Cislunar transport analysis. Her $\mathrm{PhD}$ research focuses on exploiting artificial intelligence techniques to enable trajectory design and optimization for chemical and low-thrust spacecraft in complex dynamical regimes. 


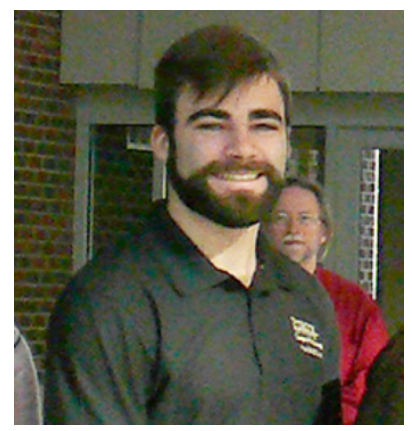

Alex Davis is currently a PhD student at CU Boulder working with Professor Dan Scheeres. His research focuses on the impact of higher order mass distribution parameters on the Full TwoBody Problem applied to estimation and simulation of binary asteroid systems. Other research interests include mission design, the three-body body problem and dynamical systems theory.

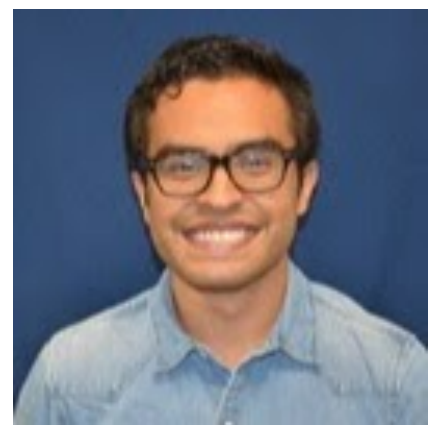

Dr. Armando Delgado is a test design and analysis engineer at NASA's John C. Stennis Space Center. He received his B.S., M.S. and Ph.D. in mechanical engineering from the University of Texas at El Paso. His dissertation focused on the production of structural materials in the moon and Mars from in-situ resources using combustion synthesis techniques.

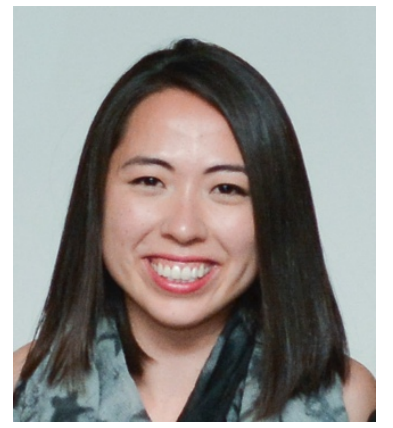

JoAnna Fulton is a PhD student at the University of Colorado at Boulder. She received her B.S. degree in Mechanical Engineering at the University of Florida and M.S. degree in Aerospace Engineering Sciences from the University of Colorado at Boulder. She is pursuing her PhD under Dr. Hanspeter Schaub in the Autonomous Vehicle Systems Lab at the Colorado Center for Astrodynamics Research. She is a Smead Scholar and an NSF Graduate Fellow. Her research interests include deployable structures, large scale space structures, and flexible, multi-body system dynamics modeling. 


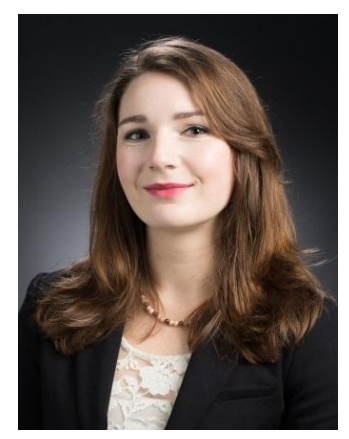

Dr. Lauren Jozwiak is a postdoctoral researcher at the Johns Hopkins University Applied Physics Laboratory. She received her B. S. in Earth, Atmospheric, and Planetary Science from MIT, and her Ph.D. in Planetary Science from Brown University. As a graduate student, she was a member of the MESSENGER MDIS instrument team and the GRAIL science team. Her research addresses volcanic processes and landforms on terrestrial bodies, focusing on magma transport processes, eruption dynamics, and landforms. Her studies utilize remotely sensed data and volcanological modeling.

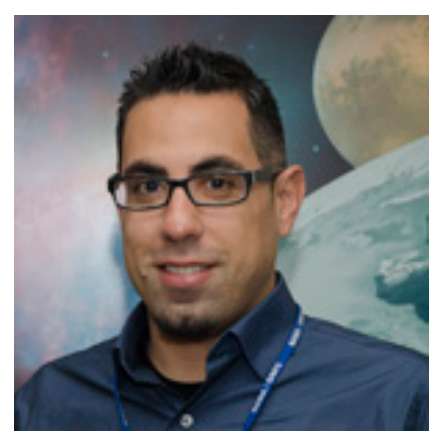

Dr. Alain Khayat is a postdoctoral research associate at NASA's Goddard Space Flight Center and the University of Maryland. He received his diplome d'ingenieur in Mechanical Engineering from the Lebanese University, and a Ph.D. in Astronomy from the University of Hawai $i$ at Manoa. His research focuses on developing radiative transfer models for the atmosphere of Mars between near-infrared and submillimeter frequencies. His ground and space based observations of Mars include the search for volcanic outgassing on the planet, mapping atmospheric $\mathrm{D} / \mathrm{H}$, and characterizing the water cycle above the Northern polar cap of Mars.

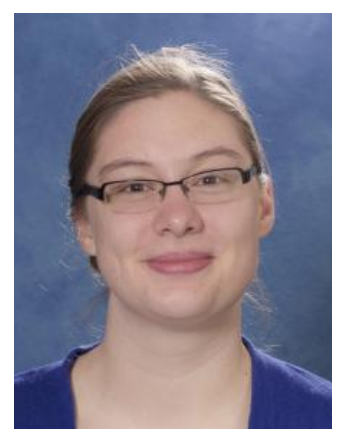

Margaret E. Landis is a Ph.D. candidate and National Science Foundation Graduate Research Fellow at the Lunar and Planetary Laboratory, University of Arizona. Her graduate work focuses on studies of water ice in the inner solar system, particularly the recent stability on Mars and Ceres. She received her B.S. in Physics and Astronomy from Northern Arizona University. She 
is a member of the High Resolution Imaging Science Experiment (HiRISE) science team and is a Guest Investigator student on Dawn.

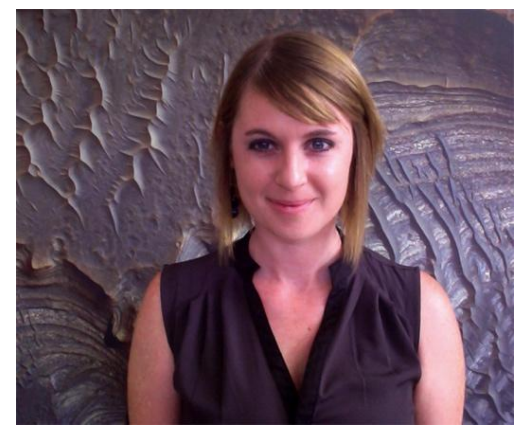

Dr. Jamie L. Molaro is a NASA Postdoctoral Program Fellow at the Jet Propulsion Laboratory, California Institute of Technology. She received her B.S. in Physics from San Francisco State University and her Ph.D. in Planetary Sciences from the University of Arizona. Her research focuses on physical weathering and fracture processes from micro- to macroscopic scales, and the role of thermal cycling on the evolution of rocky and icy airless body surfaces. She employs a combination of numerical modeling, laboratory and field studies, and analysis of remote sensing data in her work.

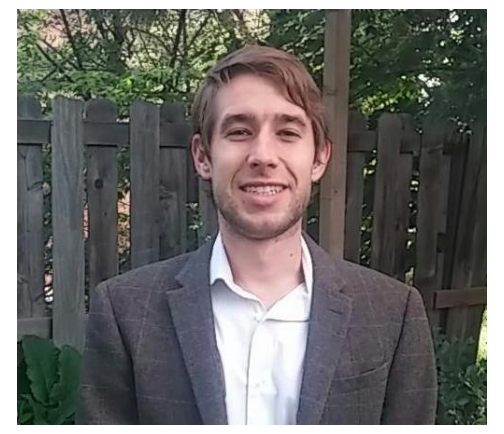

Marek Slipski is a graduate student at the University of Colorado Boulder and a member of the MAVEN science team. He earned a B.S. in physics and astronomy from the University of Rochester. His research focuses on understanding the evolution of Mars' atmosphere through isotope ratios of key atmospheric species. He has modeled the effects of important long-term processes on isotope ratios and analyzed data from MAVEN's NGIMS instrument to study the composition and structure of the upper atmosphere.

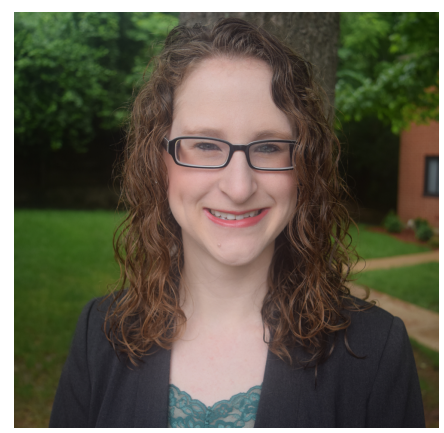

Dr. Sarah Valencia is a recent graduate of Washington University in St. Louis where she earned her Ph.D. in the department of Earth and Planetary Sciences. Prior to 
graduate school she earned her B.S. in Geological Sciences at the University of Michigan. Her work focuses on the evolution of lunar magmatic systems by studying lunar meteorites and samples from the Apollo collection. In addition, she uses data from the Lunar Reconnaissance Orbiter Camera to study evolved volcanic rocks on the lunar surface.

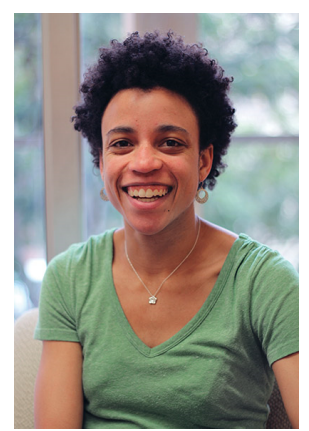

Dr. Jessica Watkins was a postdoctoral fellow in the Division of Geological and Planetary Sciences at Caltech during the Planetary Science Summer School. Her research focuses on using orbital and rover image, spectral, and topographic data to investigate the geology, stratigraphy, and geomorphology of Mars. She completed her undergraduate in Geological and Environmental Sciences at Stanford University. Her thesis work at UCLA, where she received her PhD in Geology, focused primarily on constraining the emplacement mechanisms of long-runout landslides in equatorial Mars. She is currently a member of NASA's 2017 Astronaut Candidate Class.

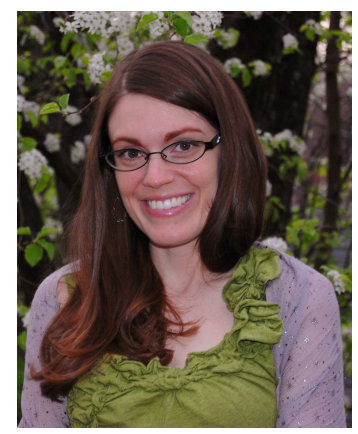

Dr. Cindy L. Young is an instrument scientist at NASA Langley Research Center. At the time the concept study for OCEANUS was completed, she was a postdoctoral researcher at the Georgia Institute of Technology investigating the surface compositions of Saturn's icy moons using mid-infrared spectroscopy. Her PhD project employed Earth satellite remote sensing data to evaluate the radiative impacts of volcanic aerosol in the Arctic. She is now a member of the Radiation Budget Instrument science team and is working on the mission concept for a follow-on radiometer to help continue the measurement record of Earth's radiation balance from space. 


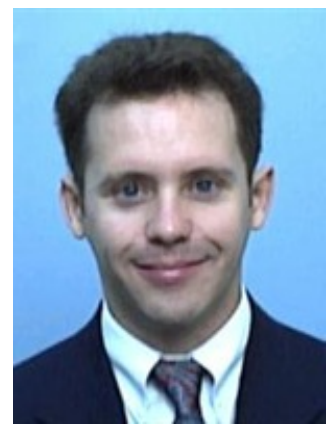

Dr. Charles J. Budney received a B.S in Geochemistry from Caltech in 1988 and his Ph.D. degree in Geology and Geophysics from the University of Hawaii at Manoa in 1997. He is a senior systems engineer at the Jet Propulsion Laboratory, California Institute of Technology, where he is currently involved in planning for future missions to Mars and working to get NASA data into the decisional processes for water use in the western U.S. He has worked at JPL for over 20 years.

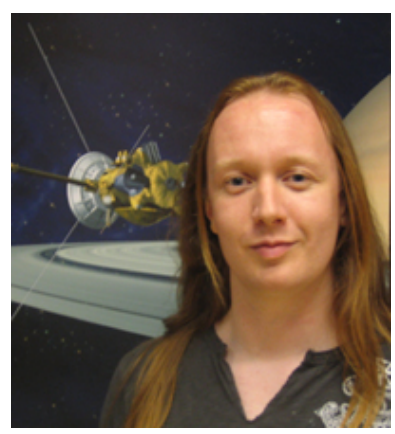

Dr. Karl L. Mitchell received a B.Sc. in Physics with Space Science and Technology from Leicester University in 1995, an M.Sc. in Remote Sensing from the University of London in 1996, and his Ph.D. in Environmental Science from Lancaster University in 2002. In 2005 he moved to Jet Propulsion Laboratory, California Institute of Technology, initially as a postdoctoral researcher working on the Cassini project, and from 2008 as a staff scientist. His research focuses primarily on geological fluid dynamics, science mission formulation and remote sensing techniques spanning multiple Solar System bodies, and he has been actively involved in the Cassini mission in a number of roles, including Cassini RADAR Team, science planning and legacy product management. 